\title{
Aplicación móvil de gestión empresarial para fincas ganaderas, articulado con el programa de trazabilidad bovina
}

Business Management App for cattle ranches, articulated with the bovine traceability program

Aplicativo móvel de gestão empresarial para fazendas de gado articulado com o programa de rastreabilidade bovina

\section{Luiyiana Pérez ${ }^{1}$ \\ Rolando Lasso ${ }^{2}$}

Recibido: septiembre 5 del 2018

Aprobado: diciembre 10 del 2018 Disponible en línea: enero 15 de 2019

Cómo citar este artículo:

L. Pérez y R. Lasso, "Aplicación móvil de gestión empresarial para fincas ganaderas, articulado con el programa de trazabilidad bovina", Revista Ingeniería

Solidaria, vol. 25, n. ${ }^{\circ} 1,2019$.

DOI: https://doi.org/10.16925/2357-6014.2019.01.10

Artículo de investigación. https://doi.org/10.16925/2357-6014.2019.01.10

1 Universidad Tecnológica de Panamá

ORCID: https://orcid.org/0000-0001-5069-4950

Correo electrónico: luiyiana.perez@utp.ac.pa

2 Universidad Tecnológica de Panamá

ORCID: https://orcid.org/0000-0002-6200-466X 


\section{Resumen}

Introducción: el artículo es producto de la investigación "Desarrollo e implementación de un software para la gestión y control de las fincas pecuarias, articulado con el programa de trazabilidad bovina", financiado por SENACYT, realizado durante 2017-2018, en la Universidad Tecnológica de Panamá, sede Azuero, con colaboración de la Consultora Luiyiana Pérez.

Problema: este proyecto surge de la ausencia directa de los productores en el registro de la información de campo, y en el programa de trazabilidad bovina.

Objetivo: desarrollar una aplicación móvil que permita administrar la gestión de las fincas ganaderas, con integración al sistema de gestión pecuario (SIGEPE) desde las agencias del Ministerio de Desarrollo Agropecuario.

Metodología: se realizó un estudio del nivel del índice de la brecha digital (IBD) en el sector, se utilizó un muestreo estratificado proporcional para la selección de los productores y se utilizó el modelo vista-controlador para el desarrollo de la aplicación móvil.

Resultado: se levantó una base de datos con información de nutrición, programa sanitario, productos veterinarios entre otros, se tiene la aplicación desarrollada con sus respectivos módulos, se tiene un plan de capacitación para la implementación de la aplicación móvil.

Conclusión: la competitividad empresarial pecuaria vs la regulación de la trazabilidad bovina demanda el registro oportuno de la información de campo en todos los puntos de producción animal.

Originalidad: la incorporación del control pecuaria por parte de las distintas agencias y su articulación con la trazabilidad bovina.

Limitaciones: las habilidades tecnológicas deficiente de los usuarios en el manejo y usos de las TIC.

Palabras clave: Complejidad creciente, estructura organizacional, sistemas de procesamiento de información.

\section{Abstract}

Introduction: The article is the result of Research "Development and implementation of a software for the management and control of livestock farms, articulated with the bovine traceability program". This initiative was financed by SENACYT and carried out at the Universidad Tecnológica de Panamá, Azuero Campus, with the collaboration of Consultant Luiyiana Pérez during 2017 and 2018

Problem: This project arises from lack of producers in the field information registration and bovine traceability program.

Objective: To develop an app that allows you to manage the management of livestock farms, integrated to Livestock Management System (SIGEPE in its Spanish acronym) from the agencies of the Ministry of Agricultural Development.

Methodology: A study of the level of the digital divide index (IBD) in the sector was carried out; a proportional stratified sampling was used for the selection of the producers and the view-controller model was used to develop the app.

Result: A database was created, with information about nutrition, health programs, veterinary products among others. An application was developed, including its respective modules and a training plan for the implementation of the app.

Conclusion: The livestock business competitiveness vs. bovine traceability regulation demands the appropriate field information registration at all points of animal production.

Originality: The incorporation of livestock control by the various agencies and their articulation with the bovine traceability. 
Limitations: The users' deficient technological skills in the management and use of ICTs.

Keywords: Growing Complexity, Organizational Structure, Information Processing Systems.

\section{Resumo}

Introdução: o artigo é produto da pesquisa "Desenvolvimento e implementação de um software para a gestão e controle das fazendas pecuárias articulado com o programa de rastreabilidade bovina", financiada por SENACYT, realizada entre 2017-2018, na Universidad Tecnológica de Panamá, sede Azuero, com colaboração da consultora Luiyiana Pérez.

Problema: este projeto surge da ausência direta dos produtores no registro da informação de campo e no programa de rastreabilidade bovina.

Objetivo: desenvolver um aplicativo móvel que permita administrar a gestão das fazendas de gado com integração ao sistema de gestão pecuária (SIGEPE) a partir das agências do Ministério de Desenvolvimento Agropecuário.

Metodologia: realizou-se um estudo do índice de desigualdade digital no setor, utilizou-se uma amostragem estratificada proporcional para a seleção dos produtores e utilizou-se um modelo-vista-controlador para o desenvolvimento do aplicativo móvel.

Resultado: levantou-se uma base de dados com informação de nutrição, programa sanitário, produtos veterinários, entre outros, há o aplicativo desenvolvido com seus respectivos módulos e um plano de capacitação para a implementação do aplicativo móvel.

Conclusão: a competitividade empresarial pecuária vs. a regulação da rastreabilidade bovina demandam o registro oportuno da informação de campo em todos os pontos de produção animal.

Originalidade: a incorporação do controle pecuário por parte das diferentes agências e sua articulação com a rastreabilidade bovina.

Limitações: as habilidades tecnológicas deficientes dos usuários no uso das TIC.

Palavras-chave: Complexidade crescente, estrutura organizacional, sistemas de processamento de informação.

\section{Introducción}

Todo negocio será sostenible y competitivo si es capaz de administrar su información, y esto será posible con el uso de las Tecnología de Información y Comunicación (TIC) [1], [2], [3].

Los teléfonos móviles han conferido más poder a la población y han alentado el espíritu empresarial en los países en desarrollo [4]. La utilización de la tecnología móvil en el proyecto se da debido a que la mayoría de las fincas pecuarias de la provincia de Los Santos se encuentran en comunidades rurales, y además, la tecnología móvil es un medio económico y accesible de comunicación muy utilizados por el sector.

Según el estudio realizado [5] sobre el índice de la brecha digital en el sector pecuario en la provincia de Los Santos, nos dice que el $97 \%$ de los productores 
cuentan con tecnología móvil, es una tecnología que permite crear oportunidades económicas y fortalece el intercambio de información [4]. La tecnología móvil es un instrumento ideal para la inclusión financiera del negocio pecuario, por su crecimiento constante en estas últimas décadas, esto por un lado y por otro se tiene la capacidad para almacenar datos, sin necesidad de estar conectados directamente al servidor central, gracias a la base de datos SQLite que tiene incorporado. SQLite atiende a la problemática del acceso continuo a internet, que tiene el $87 \%$ de las fincas en la provincia, por su ubicación rural, esto permite la sincronización de los datos una vez se conecte a la red.

Este proyecto surge de la ausencia directa de los productores en el registro de la información de campo, y en el programa de trazabilidad bovina que actualmente se implementa en el país, La trazabilidad bovina es la capacidad para seguir el movimiento de un animal a través de etapa(s) especificada(s) de la producción, transformación y distribución. Un sistema de trazabilidad holístico incluye, como mínimo, elementos de identificación, bases de datos y un flujo de información [6]. La información de campo es la fuente primaria para alimenta cualquier sistema de trazabilidad bovina y consiste en: registro de las generales de la finca, composición ganadera o inventario, reproducción, producción, nutrición, programa sanitario de los animales, comercialización, etc.

Las pymes (pequeñas y medianas empresas) pecuarias, son las beneficiarias directas del proyecto, al incorporar el uso de las Tecnologías de Información y Comunicación (TIC) en la gestión de información empresarial, y a medida de que se logre la igualdad de oportunidades, mejore la calidad de vida de los ciudadanos, el bienestar social, y se disminuya con el índice de brecha digital de 5,25 que presentan las mismas, se proyectan futuros proyectos en el área de minería de datos e inteligencia de negocio.

El proyecto consiste en desarrollar e implementar una aplicación móvil para pequeños y medianos productores de ganado vacuno articulado con el programa de trazabilidad bovina, cuya implementación de validación será efectuada en la provincia de Los Santos, y después de esta etapa estará a disposición de cualquier productor a nivel nacional.

\section{Revisión de literatura}

En noviembre de 2013, el Gobierno panameño promulga la ley No. 104, para regular el Programa Nacional de Trazabilidad o Rastreabilidad Pecuario, en el cual, se establece los lineamientos y las directrices para preservar la inocuidad de los alimentos de origen animal de consumo nacional y de exportación. En 2015 se inicia el desarrollo 
de la aplicación de trazabilidad bovina en Panamá bajo la responsabilidad de OIRSA (Organismo Internacional Regional de Sanidad Agropecuaria), con una proyección de cinco años, iniciando con el registro de los establecimientos o fincas, los proveedores y la identificación de los animales, después de los primeros meses de nacido, a través de los formularios F-TZ01 y F-TZ02. Se han capacitado a operadores externos para el levantamiento de los datos, y se están colocando los aretes a los animales bovinos para su identificación individual; en 2017 se decide continuar el proceso por zonas y se inicia con la Zona 1: Bocas del Toro y Chiriquí, para luego pasar a la Zona 2 de Herrera y los Santos.

El proceso de trazabilidad que se está implementando en Panamá abarca la trazabilidad grupal, la trazabilidad individual y la trazabilidad de proceso.

Para el año 2015, tras analizar el proceso y el estudio del concepto de trazabilidad bovina, las características culturales y empresariales de las pymes pecuarias en Panamá, la existencia de un nivel de 5,2 del índice de la brecha digital en el sector [5] y la necesidad de las TIC como ente fundamental para la implementación exitosa del proceso, se desarrolla la tesis doctoral titulada [5] Modelo de Arquitectura Empresarial (AE) como instrumento para gestionar la trazabilidad vacuna en las pymes pecuarias en Panamá, Provincia de los Santos, en el cual se establece la rutas y los componentes fundamentales de las arquitectura del negocio, y la arquitectura de Tecnología de Información (TI), conformada esta última por la arquitectura de datos, de aplicaciones y de infraestructura de comunicación. Se hace énfasis que en el proceso que se está implementando en el país hace falta un componente fundamental, la incorporación del sector empresarial pecuario (ver figura 1), como fuente primaria de datos, y se selecciona del portafolio del modelo de AE el proyecto "Desarrollo del software para la gestión y control de las fincas pecuarias, articulado con el programa de trazabilidad bovina", el cual, recibe financiamiento por la SENACYT en la categoría Innovación en el Sector Agropecuario, cuyo avances se presentan en este documento.

Los datos obtenidos en esta investigación reflejan que las pymes productoras de ganado vacuno cuentan con un bajo nivel de gestión de la información del negocio, debido principalmente a su poco interés por la información, su baja valorización de la misma, y su pobre desarrollo empresarial y tecnológico, es una realidad a la que se tiene que enfrentar, por eso se ha considerado trabajar con productores con un grado de sensibilización al cambio tecnológico. Según estudios realizados por Kebebe [7], nos presenta una realidad muy parecida y coincidimos en que "Las funciones de sistemas de innovación débiles o faltantes, como el espíritu empresarial, la difusión del conocimiento, el desarrollo del mercado y la promoción de políticas, reflejan la baja aceptación de tecnologías mejoradas en el sector ganadero". 


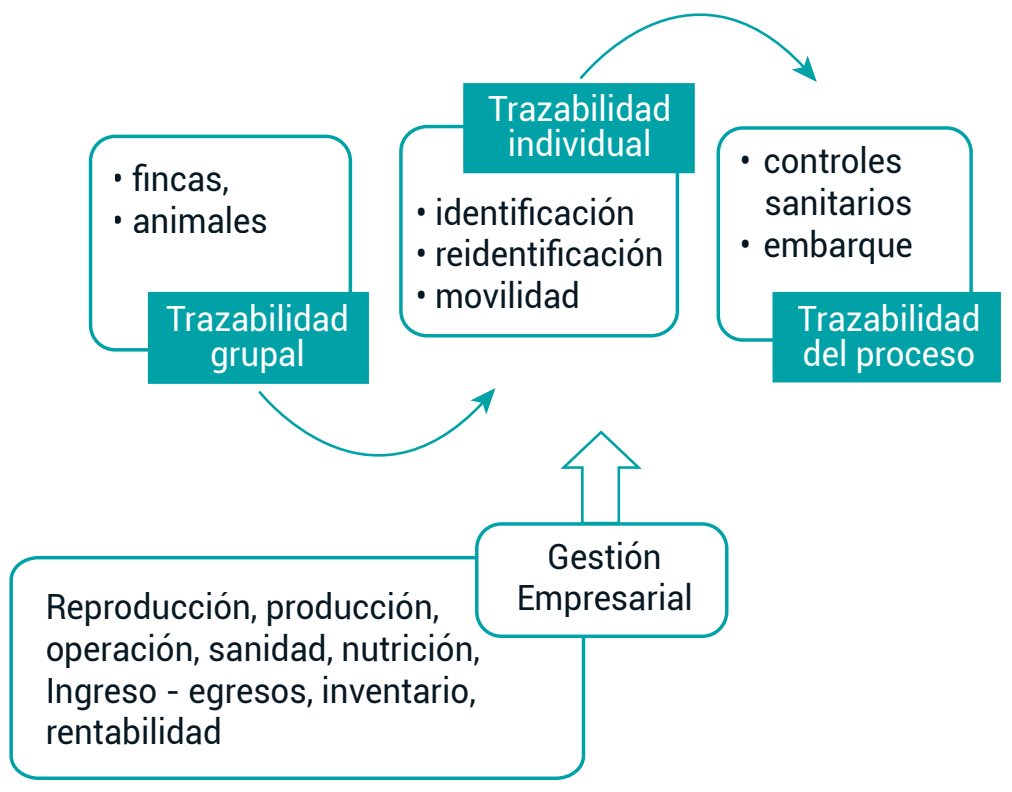

Figura 1. Propuesta del proyecto

Fuente: elaboración propia, con base en [9].

No podemos obviar que la situación del poco desarrollo empresarial y tecnológico del sector ganadero obedece a la escasez de proveedores nacionales de insumos y servicios tecnológicos clave, mercados de insumos y productos débiles y una interacción precaria entre los actores de la cadena de valor han sido los principales obstáculos para el desarrollo de las fincas ganaderas [7].

\section{Metodología}

El proyecto se caracteriza por ser una investigación aplicada, ya que se generan conocimientos dirigidos al sector productivo, con el fin de mejorarlo y hacerlo más eficiente.

Se desarrollaron dos aplicaciones o interfaces: una aplicación de escritorio para los administradores del MIDA (Ministerio de Desarrollo Agropecuario), denominado Sistema de Información de Gestión Pecuaria (SIGEPE) y la otra es una aplicación móvil para los productores, Sistema de Información Empresarial de Fincas Ganaderas, SIEFIGAN, producto que se describe en este documento. 


\section{Métodos}

\subsection{Calculo del nivel de la brecha digital en el sector}

Para determinar el tipo de tecnología a utilizar y los diseños de la aplicación, se consideraron los resultados del estudio sobre el nivel de índice de la brecha digital en el sector pecuario, se utilizó la ecuación para el cálculo del acceso digital de la Unión Internacional de Telecomunicación UIT.

$$
\mathrm{IAD}=[(\mathrm{SA} \times \mathrm{W} 1)+(\mathrm{SU} \times \mathrm{W} 2)+(\mathrm{SC} \times \mathrm{W} 3)+(\mathrm{SE} \times \mathrm{W} 4]
$$

Donde:

W1: 0,40 representando el ponderado para el componente de acceso.

W234 $=0,20$ representando el ponderado para el resto de los componentes.

El índice de la brecha digital (IBD) se estimaría a través del cálculo del IAD con la siguiente ecuación:

$$
\mathrm{IBD}=10-\mathrm{IAD}
$$

\subsection{Fases de desarrollo}

Para el desarrollo de la aplicación se utilizó las fases de un ciclo de desarrollo de software: análisis, diseño, desarrollo y pruebas, está última se dividió en dos partes, por lo complejo de la implementación.

Durante la fase de análisis se entrevistaron a productores y colaboradores de las distintas agencias de la regional de Los Santos, la cual permitió la recolección de los requerimientos y su clasificación en tres partes: el entorno, el usuario y la funcionalidad.

En el análisis del entorno (1) se determinaron las características técnicas del dispositivo móvil como: sistema operativo, infraestructura para la transferencia de la información, el manejador de base de datos a nivel de servidor, y base de datos embebida. En el análisis del usuario (2) se definió la interacción del productor, la gama tecnológica de los teléfonos móviles y los usuarios a la que va dirigido, para el caso en estudio, los productores tienen baja o nula habilidad en el manejo y uso de las TIC. Con el análisis de la funcionalidad de la aplicación (3) se identificaron las tareas o procesos que debe realizar la misma. 
En la fase de diseño se realizaron dos actividades: definición de escenario y estructuración del software.

Las aplicaciones móviles se pueden diseñar para ejecutarse en diferentes escenarios, dependiendo del sistema operativo, de la conexión y sincronización con el servidor o aplicación central. Para el proyecto se seleccionó el sistema operativo Android, por ser de código abierto, por su adaptabilidad a distintos dispositivos móviles, y su funcionalidad con SQLite, ya que esta permite que una vez instalada la aplicación pueda funcionar desconectada con el servidor central, es decir, los procesos se pueden realizar en el dispositivo móvil y una vez conectado o sincronizado con el servidor, los datos son cargados al mismo, utilizando el protocolo de transferencia de hipertexto (HTTP).

En la actividad de estructuración del software se utilizó el diagrama de clases para el modelo de lenguaje unificado, y se estableció el diseño estructural, a través del modelo vista controlador, en donde el modelo contiene una representación de los datos que maneja el sistema, su lógica y sus mecanismos de persistencia; la vista compone la información que se envía al cliente y los mecanismos de interacción hombre-computador; y el controlador, que actúa como intermediario entre el modelo y la vista, gestiona el flujo de información entre ellos y las transformaciones para adaptar los datos a las necesidades de cada uno. Se utilizó el framework Laravel para la codificación, a continuación, se presenta uno de los modelos, utilizando el diagrama de clase [10].

$$
\begin{aligned}
& {[\text { Animal] }<>-\star>\text { [Eventos] }} \\
& {[\text { Eventos }]^{\wedge}-[\text { Reproducción] }} \\
& {[\text { Eventos }]^{\wedge}-[\text { Nutrición] }} \\
& {[\text { Eventos }]^{\wedge}-[\text { Producción] }} \\
& {[\text { Eventos }]^{\wedge}-[\text { ControlSanitario] }}
\end{aligned}
$$

Esquematizando esta primera parte del diagrama en la figura 3.

$$
\begin{aligned}
& \text { [Reproducción] ^^-[Celo] } \\
& \text { [Reproducción] }{ }^{\wedge} \text {-[Semental] } \\
& \text { [Reproducción] }{ }^{\wedge} \text {-[Parto] } \\
& \text { [Reproducción] ^^-[Crecimiento] } \\
& \text { [Nutrición] ^^-[Pasto] } \\
& \text { [Nutrición] }{ }^{\wedge}-[\text { Concentrado] } \\
& \text { [Nutrición] ^^-[sal] }
\end{aligned}
$$



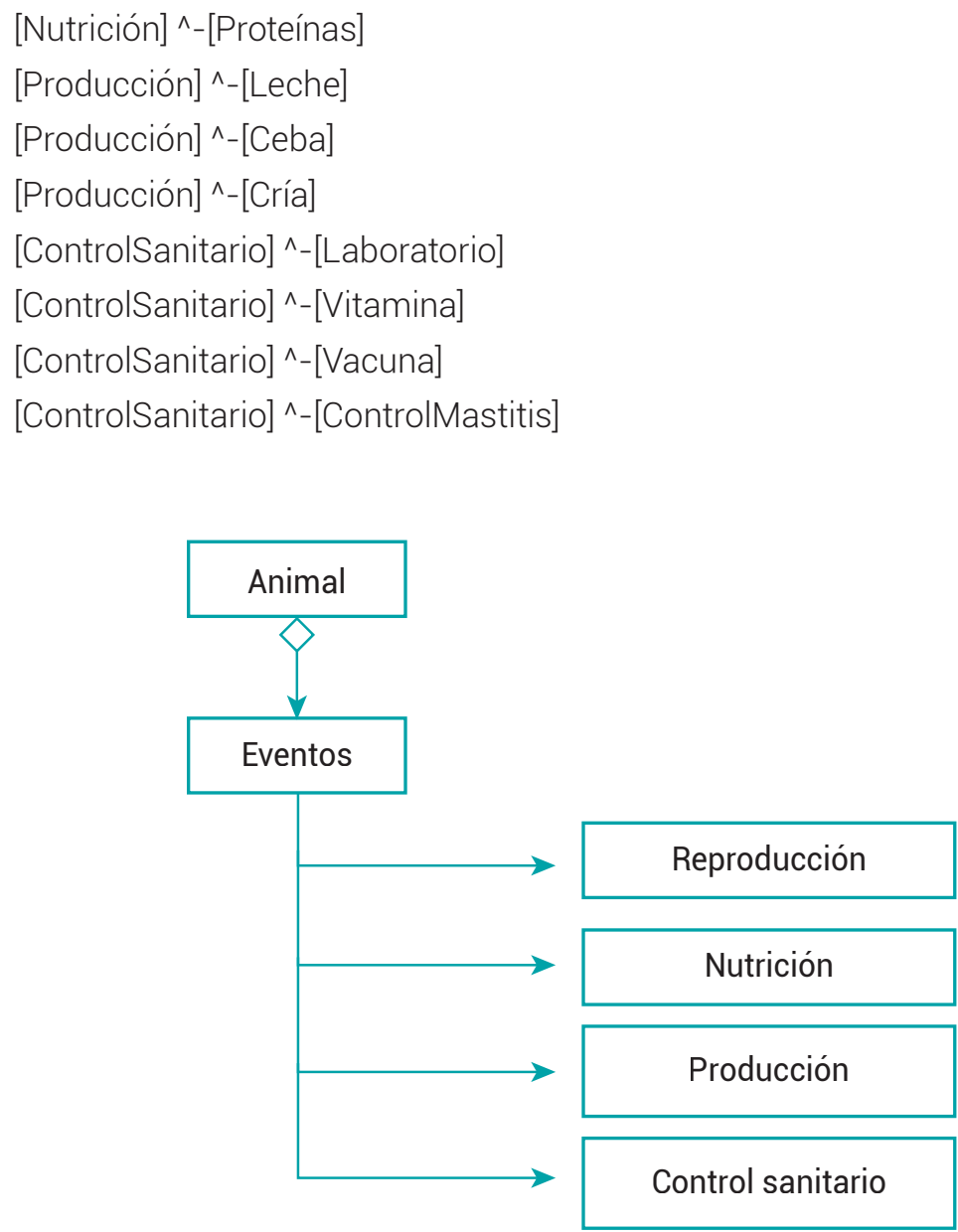

Figura 2. Diagrama de Clase UML, clase Animal Fuente: elaboración propia.

Fase de desarrollo: en esta fase se realizaron las actividades de la codificación de los módulos, se utilizó XML para el desarrollo de las vistas o fragmentos, el lenguaje Java para la programación de los controladores y el framework Laravel para la comunicación con el controlador API de la base de datos PostgreSQL y las funciones de las sentencias SQL, las cuales fueron desarrolladas en PHP de cada controlador de los objetos o modelos en el servidor central.

Se realizaron las pruebas unitarias de cada elemento desarrollado: objeto, fragmento, actividad, vista entre otros, antes de ponerlo a funcionar con el resto de los otros elementos y luego se comprobó la integración entre ellos, se verificó si los resultados eran los esperados. 
La fase de prueba en la parte I se inició con el registro de la información en libros de un productor correspondiente al periodo de 2013-2018, para recoger un historial de reproducción y producción, esto permitió validar si los informes e indicadores generados correspondían a los requeridos; una vez validado, se procedió a realizar los ajustes de programación correspondiente.

La fase de prueba parte II, planificada en fecha posterior de este escrito: corresponderá a la prueba de campo con los productores, esta iniciará con la capacitación sobre el manejo del aplicativo y luego a cada productor se le asignará un supervisor del MIDA para que verifique si la información introducida por el productor es válida, así como su apoyo técnico en el manejo correcto de la aplicación móvil. Igualmente, se validará la funcionalidad e integración de las dos aplicaciones que abarca el proyecto en totalidad.

Es importante realizar pruebas de campo con dispositivos reales para medir el desempeño y el rendimiento de la aplicación, y corregir las fallas encontradas durante el proceso, antes del cierre final de proyecto.

\subsection{Población}

El estudio se realizó en la provincia de Los Santos, ya que allí se encuentran la mayor cantidad de pymes pecuarias del país, es decir, productores con menos de 50 hectáreas y con un máximo de 50 animales, cuyos ingresos son para su subsistencia.

La selección de los productores del sector pecuario se debió a su escaso desarrollo en lo que respecta a la utilización de las TIC para la gestión de la información desde la finca hasta la trazabilidad individual. Esto representa una situación de negocio compleja que plantea requerimientos de cambio al mejor costo y efectividad posible. Por lo tanto, la principal razón para desarrollar una aplicación móvil para la gestión empresarial pecuaria es facilitar el acceso y la sincronización de los datos.

Los criterios considerados para la selección de la muestra en la implementación del software, fue el muestreo estratificado proporcional de los productores perteneciente a las ocho agencias (estratos) del MIDA de la regional de Los Santos, atendiendo a la siguiente ecuación.

$$
n=\frac{z_{a}^{2} \times p \times q}{e^{2}} \Rightarrow n c=\frac{n}{1+n / N}
$$


En donde:

$\mathrm{Z}$ = es la desviación del valor medio que se acepta para logra el nivel de confianza deseado, 95\%.

p = es la proporción estimada de la población que cumple con la característica deseada (pertenecen al programa de difusión tecnológica).

$\mathrm{e}=$ es el margen de error aceptado, $5 \%$.

$\mathrm{n}=$ tamaño de la muestra para toda la población, 60 productores.

$q=1-p$.

ne $_{\mathrm{i}}=$ tamaño de la muestra para el estrato $\mathrm{i}$.

$\mathrm{n}_{\mathrm{i}}=$ población de cada estrato.

$\mathrm{N}=$ total de la población, 70 productores.

\subsection{Herramientas}

Las herramientas utilizadas para el desarrollo de la app son: Android studio, SQLite, Java, el framework Laravel, PHP y PostgreSQL, sistemas operativos: Andrid, Centos.

\section{Resultados}

Los resultados en este proyecto están en función de las fases de desarrollo descritas en el acápite de la metodología:

En la fase de análisis se visitaron las fincas y las diferentes agencias de la regional de Los Santos, se definieron las entradas y salidas de datos, los roles de usuarios, los módulos a desarrollar y los procesos del flujo de la información.

En la fase de diseño de la aplicación (ver figura 4):

- Diseño de la base de datos: en esta actividad se confeccionaron las tablas con sus campos, claves primarias y secundarias y sus relaciones, de acuerdo a los lineamientos de la fase I, con un total de 93 tablas, siguiendo el modelo relacional.

- Diseño de las interfaces de la aplicación: en esta etapa se confeccionó la plantilla de la interfaz de la aplicación móvil, con la respectiva distribución de los menús, submenú, encabezado, pie de página y contenido.

- Diseño del marco de trabajo (framework) con los modelos, controladores y la estructura de las vistas.

- Diseño de las pantallas de entrada: durante esta actividad se diseñaron los formularios o vistas de entrada para alimentar las diferentes tablas de la 
base de datos, lo que estandarizó el flujo de la información y el comportamiento de las acciones (consulta, registro, actualización).

La estructuración de los menús de la aplicación móvil está representada por imágenes en el centro de la pantalla, una vez se entra haciendo clic a una opción, la información se presentará de arriba hacia abajo, siguiente una jerarquía de proceso, como se muestra en la figura 4.

La vista de entrada de la aplicación tiene un formato estándar para el flujo de la información, estructurada de arriba hacia abajo, la mayoría de los datos vendrán almacenados previamente para su selección, tratando que el productor escriba lo menos posible como se muestra en la figura 5.

El desarrollo de los distintos fragmentos en el Android Studio se programó en el lenguaje Java, se desarrolló una interfaz o autenticación mediante token y se desarrollaron todas las funciones de la API (Application Programming Interface) en PHP para la comunicación de la aplicación Android con el servidor Central, sincronizando los datos de SQLite al servidor de base de datos PostgreSQL. El servidor central trabaja con el framework Laravel en donde se encuentra el controlador con todas las funciones SQL. La metodología de programación utilizada fue la programación orientada a objeto, y el modelo vista-controlador.

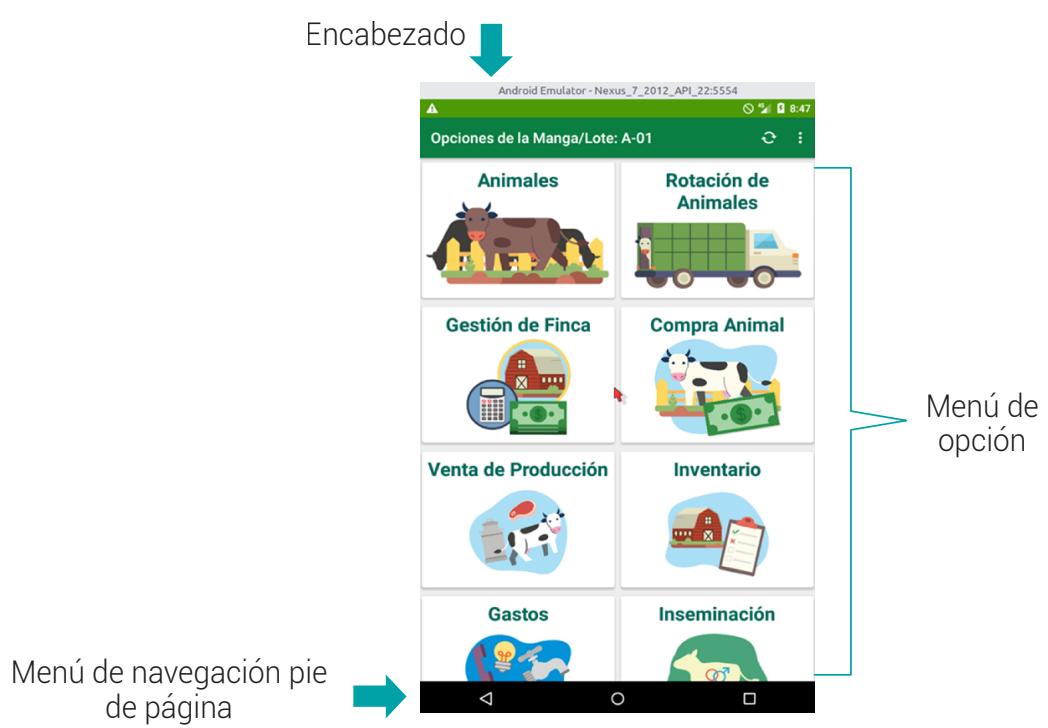

Figura 4. Pantalla del menú de opciones la aplicación móvil Fuente: elaboración propia. 


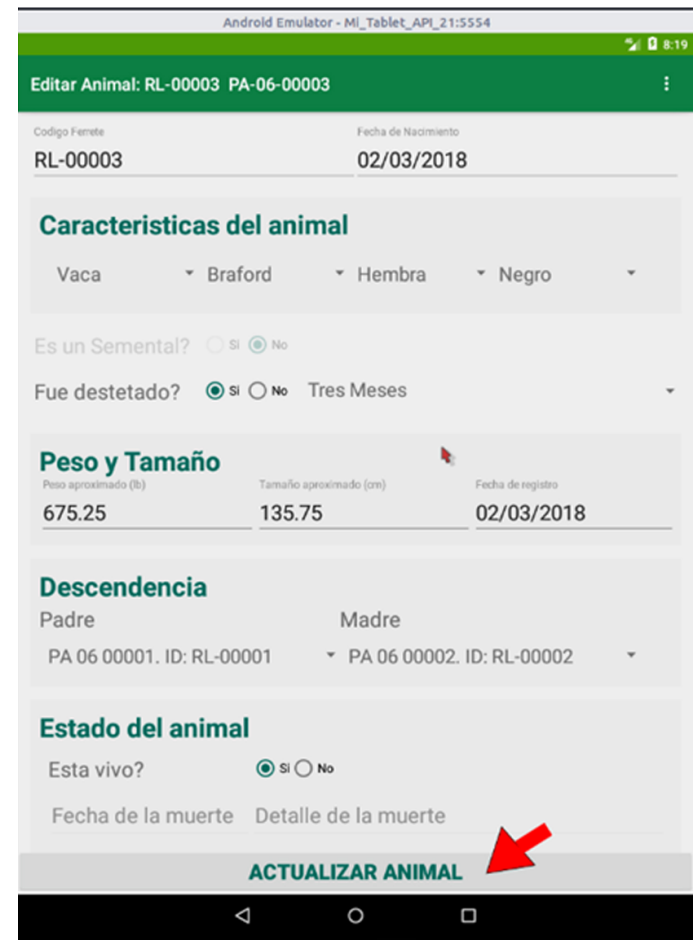

Figura 5. Pantalla o vista de entrada de un animal. Aplicación móvil Fuente: elaboración propia.

\section{Discusión}

La competitividad de la organización empresarial pecuaria y la regulación de la trazabilidad bovina demanda el registro oportuno de la información de campo en todos los puntos de producción animal, para entrar en un proceso regulatorio sanitario y garantizar la seguridad alimentaria de los ciudadanos. La trazabilidad de las enfermedades/plagas e insumos son los elementos que aumentan la confianza de los consumidores en la seguridad de los alimentos, según resultados obtenidos en el estudio "An integration of traceability elements and their impact in consumer's trust" [8].

Trabajar con productores sensibilizados permite implementar el proyecto más rápidamente, pero su éxito dependerá de los resultados obtenidos a largo plazo, cuando se mida la rentabilidad y operatividad del negocio con el manejo de la información, durante todo el proceso de reproducción, nacimiento, crecimiento, producción y comercialización del producto animal.

Si se logra tener un banco de datos de un animal con todo su proceso de nutrición, controles sanitarios, movilidad, reproducción, etc., se puede decir entonces, que 
la trazabilidad bovina se ha implementado correctamente, se saber el historial de un animal, en cualquier momento, a través de su identificador único, se estará hablando entonces de un expediente de salud de un animal [8].

Este proyecto constituye un paso fundamental para disminuir la brecha digital media que presenta el sector, pero su disminución total dependerá de la disposición de los productores en asumir el cambio y empoderarse de las TIC como herramienta primordial en el manejo y divulgación de la información.

Se puede lograr el cambio a la medida que se implemente proyecto de capacitación continuo a lo largo de este proceso que inicia, pero no termina, ya que la reproducción es un proceso cíclico, hasta que el animal muera.

\section{Conclusiones}

El proyecto es fuente de datos primaria para el programa de trazabilidad bovina del MIDA, y la diferencia con otros proyectos del mercado es la incorporación del control pecuaria por parte de las distintas agencias.

En el diseño se ha considerado la interrelación hombre-máquina, de acuerdo a las características de los productores identificadas en el estudio del nivel del índice de la brecha digital, se busca la usabilidad, accesibilidad y funcionabilidad de la aplicación.

El levantamiento de datos fijos ayudó a proporcionar una herramienta amigable, para que el productor tenga que escribir lo menos posible, tratando de minimizar errores de captación de datos, y la estandarización de la información.

\section{Referencias}

[1] I. Ávalos, Aproximación a la gerencia de tecnología en la empresa, Caracas: Ediciones IESA, 1992. Liney Manjarrez Enriquez, Jaider Vega Jurado

[2] L. Manjarrez, y J. Vega, La gestión de la innovación en la empresa: evolución de su campo de estudio, Dimensión Empresarial, ISSN-e 1692-8563, Vol. 10, Nº. 1, 2012, págs. 18-29.

[3] G. Gaynor, Manual de Gestión en Tecnología, Bogotá: Mc Graw Hill/Interamericana de Colombia, 1999.

[4] L. Moreno. (2011). "Telefonía móvil en áreas rurales perspectiva de América Latina y el Caribe", E-agriculture.org, [En línea] Disponible en: http://www.fao.org/docrep/017/aq001s/aq001s. pdf 
[5] L. Pérez, R Miguelena y A. Diallo, "Estrategia de innovación de la arquitectura empresarial: una alternativa para la gestión de la trazabilidad del ganado vacuno en las pymes pecuarias de Panamá", Revista I+D, vol. 13, no. 1pp 54-64, 2017.

[6] Hongwu et al., "Traceability technologies for farm animals and their products in China", Food Control, vol. 79, pp. 35-43, sept. 2017, doi: https://doi.org/10.1016/j.foodcont.2017.02.040

[7] E. Kebebe, "Bridging technology adoption gaps in livestock sector in Ethiopia: A innovation system perspective", Technology in Society. [Disponible en línea en diciembre de 2018, en prensa], doi: https://doi.org/10.1016/j.techsoc.2018.12.002

[8] D. E. Matzembacher et al., "An integration of traceability elements and their impact in consumer's trust". Food Control, vol. 92, pp. 420-429 Oct. 2018, doi: https://doi.org/10.1016/j. foodcont.2018.05.014

[9] L. Pérez, R. Miguelenay A. Diallo, "Arquitectura Empresarial, una estratégia para las pymes pecuarias en Panamá", en 15th LACCEI International Multi-Conference for Engineering, Education, and Technology: "Global Partnerships for development and engineering education", Florida, Estados Unidos, jul. 2017.

[10] P, Luiyiana. M, Ramfis, y D, Abdoulaye, "Framework para la implementación de un proyecto de la arquitectura de aplicación del modelo arquitectura empresarial para la gestión de la trazabilidad pecuaria”, en VI Congreso de Ingeniería, Ciencias y Tecnología (ESTEC 2017) "Tendencias y Desafíos en Ingeniería, Ciencias y Tecnología”, oct. 2017. [En línea]. Disponible en: https://knepublishing.com/index.php/KnE-Engineering/article/view/1502 